\title{
Research and Application of the Hardware and the Sequence Control System for Chemical Water Treatment System
}

\author{
Lin Bo ${ }^{1, a}$, Sui Wei-yan ${ }^{2, b}$ and Gao song ${ }^{1, c}$ \\ ${ }^{1}$ State Grid Shandong Electric Power Research Institute, Jinan, China \\ ${ }^{2}$ Shandong University, Jinan, China \\ aurzuzu7@163.com, ${ }^{\mathrm{b}} 267265909 @ q q . c o m,{ }^{\mathrm{c}} 55703950 @ q q . c o m$
}

\begin{abstract}
Keywords: Power Plant; Chemical Water Treatment System; Programmable Logic Controller; Distributed Control System; Protection Logic; Sequential Control Logic

Abstract. This paper introduces the method of controlling system of chemical water treatment control system of thermal power plant, which is transformed into a distributed control system. According to the working principle and structure characteristics of the equipment, the protection and the sequence control logic of the system, such as filtration system, reverse osmosis, fresh water tank, acid and alkali system, were redesigned. After reformation, the water quality requirement of power plant is met, and the stability of the system is further improved.
\end{abstract}

\section{Introduction}

Chemical water treatment system is an important part of power plant auxiliary system. Its operation is related to the safety of the boiler and the continuity of production. So it affects the safety and service life of the whole power plant. As the development of chemical water treatment process, the complexity of the system is becoming more and more high. The system is also more and more strict with the requirements of automatic control.

The automatic control system of chemical water treatment for general power plant is used to PLC (Programmable Logic Controller) based. The use of PLC in the earlier power plant was longer, and the stability of the system decreased [1]. Maintenance personnel are no longer familiar with the logic of the maintenance. With the PLC transformed into DCS, chemical water treatment system is easier to realize the automation control, state monitoring, data acquisition, real time alarming, statistical printing and other functions. Compared with the PLC, DCS has the advantages of ease of use, flexibility, etc.

\section{Hardware modification}

\section{Chemical water treatment equipment}

The chemical water treatment equipment in this power plant mainly includes that three cation exchangers, three anion exchanger, three mix ion-exchangers, seven mechanical filters, four reverse osmosis units, four adding acid and alkali devices, two clarified water pumps, four fresh water pumps, three desalted pumps, four removal carbon dioxide fan, cation/anion regeneration pumps and other such devices.

\section{PLC}

The operation of the PLC system is made up of two PC, which sends out the start signal, manual/automatic control, remote manual operation and other such operations. System operating parameter setting, data, alarm are realized in the computer.

PLC cabinet: System has four PLC cabinets. The system mainly includes a set of QUANTUM PLC and isolated relays for I/O. It is used to process the system program and sends the results to the control object by the isolated relays. PLC has four I/O stations, each including a number of different types of I/O modules.

\section{DCS}

The use of DCS for transformation is Xinhua XDPS-400+. This is a series of industrial information technology, which integrates computer, network, database and automatic control technology. It can 
form a variety of independent control system, distributed control system, monitoring and data acquisition system (SCADA). It can also meet the needs of process control and information management in various industries. The system has modular design, reasonable software and hardware configuration and the ability of easy expansion. It can be widely used in large, medium and small power plant.

XDPS-400+ control unit, communication network, human machine interface station have open system architecture, the most advanced computer software and hardware. Various types of stations are redundant TCP/IP network nodes. With the configuration, the system achieves Process control and information management for power plant and other industrial processes. The system has the functions of all process input, output, collection, processing, monitoring, control, alarm, trend, record and statistical analysis. The system can also be conveniently arranged, the configuration screen and the timely addition of text annotation, so that the design and configuration of a complete. Generate configuration files can be directly download, which do not need to compile. The system can directly upload to restore graphics files, in order to easily operate and manage.

\section{Modification process}

Through the analysis of the PLC system point directory, We the establish DCS system database and determine the number and type of the required number of cards. In the modification process, we need to continue to use the original cable to save costs. According to the cable length and the position of the DCS cabinet, We re adjust the order of DCS Cards. Through the investigation of the cable, we mark the location of the wiring.

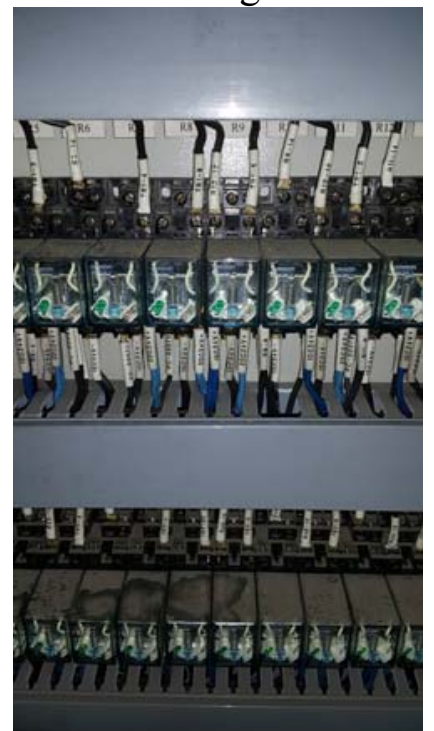

PLC

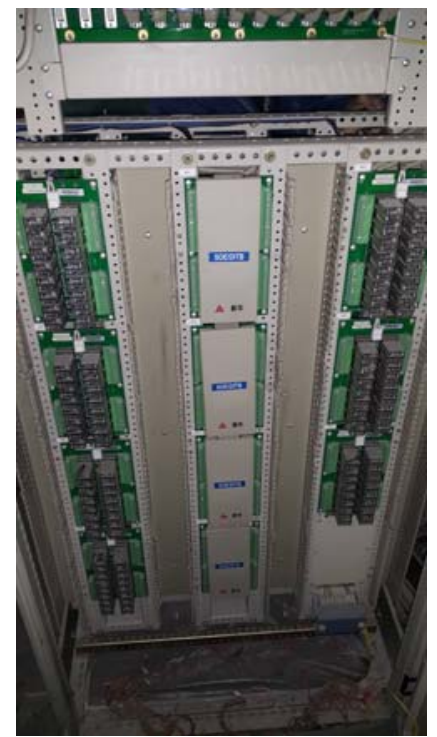

DCS

Fig.1. Before and after comparison

\section{The Sequence Control System}

After the completion of the hardware modification, we did not copy the original PLC logic. In order to achieve the function and easy operation for the purpose, we redesigned the DCS control logic. mainly includes that the sequence control of filtration system, The sequence control logic is used in filtration system, reverse osmosis system and desalted system. The protection logic is used in fresh water pump, acid and alkali system and so on [2,3]. There is little difference in the logic of these systems. Therefore this paper chooses a mechanical filter as an example and introduce its back flushing sequence control logic.

\section{Mechanical filter}

Mechanical filter is also known as pressure filter. Under certain pressure, the suspended solids in water can be filtered by the filter media. At the same time, the smelling and chlorine in water are also removed so as to achieve the purpose of purification [4]. When a certain amount of raw water is purified, the filtering medium is cleaned and washed so as to make filtering function restore. 


\section{Check the device before starting}

(1) The water level of $400 \mathrm{~m} 3$ reservoir is normal. The fresh water pump inlet and outlet manual door are open.

(2) The mechanical filter is in good condition.

(3) Compressed air system is in good condition. Instrument air pressure $\geq 0.4 \mathrm{MPa}$.

(4) All hand doors, pneumatic doors and electric doors are movement reliable

(5) A variety of instruments is put into operation or standby state.

(6) Control and adjustment device are in good condition.

(7) Control cabinet and solenoid valve have been worked.

\section{Back flushing sequence control}

Back flushing sequence control process is divided into drainage, air bump rinse, back flushing and wash. Specific circumstances are shown in Table 1.

Table 1. Back flushing process

\begin{tabular}{|l|l|c|c|c|}
\hline \multicolumn{1}{|c|}{} & \multicolumn{3}{|c|}{ process } \\
\hline inlet water valve & drainage & air bump rinse & back flushing & \multicolumn{1}{|c|}{ wash } \\
\hline outlet water valve & & & & \\
\hline back flushing inlet water valve & & & $\sqrt{ }$ & \\
\hline back flushing outlet water valve & & $\sqrt{ }$ & \\
\hline inlet valve & & $\sqrt{ }$ & & \\
\hline vent valve & & $\sqrt{ }$ & $\sqrt{ }$ & $\sqrt{ }$ \\
\hline wash outlet water valve & $\sqrt{ }$ & & & $\sqrt{ }$ \\
\hline
\end{tabular}

\section{Control system design}

The manual operator of mechanical filter back flushing sequence control is shown in Figure 2 . Automatic button is the four process control of the start button. When the sequence control problems occur, the reset button reset all STEP modules. Along the control every step, the picture will have the corresponding instruction. The drainage button is also designed as a separate operation, in order to run the corresponding sequence of steps. This will facilitate the operation for the operator.

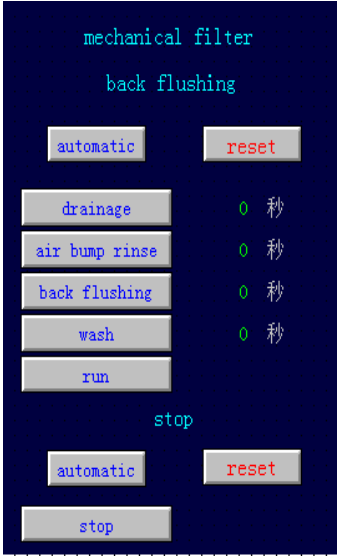

Fig.2. Manual operator

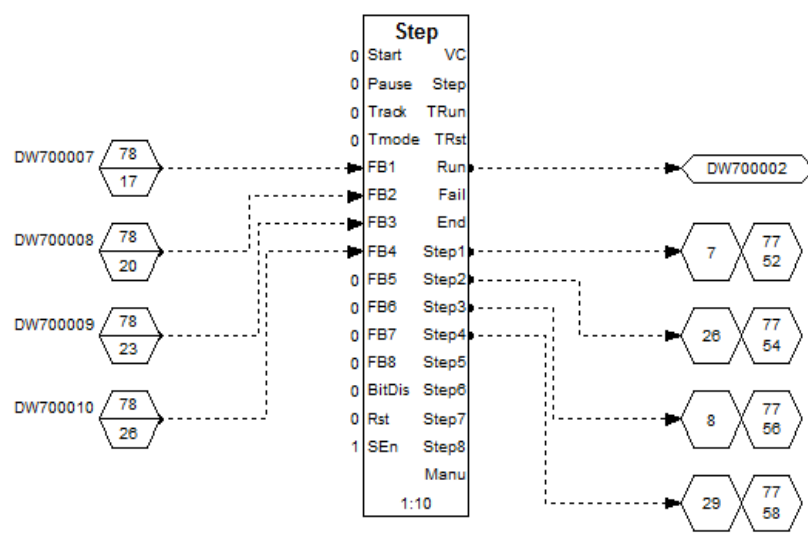

Fig.3. General logic

Back flushing sequence control start through the general logic. From step1 to Step4, the logic control valve switch and the start and stop of the pump [5]. When the feedback is correct, the logic is to enter the next step until complete sequence control. 


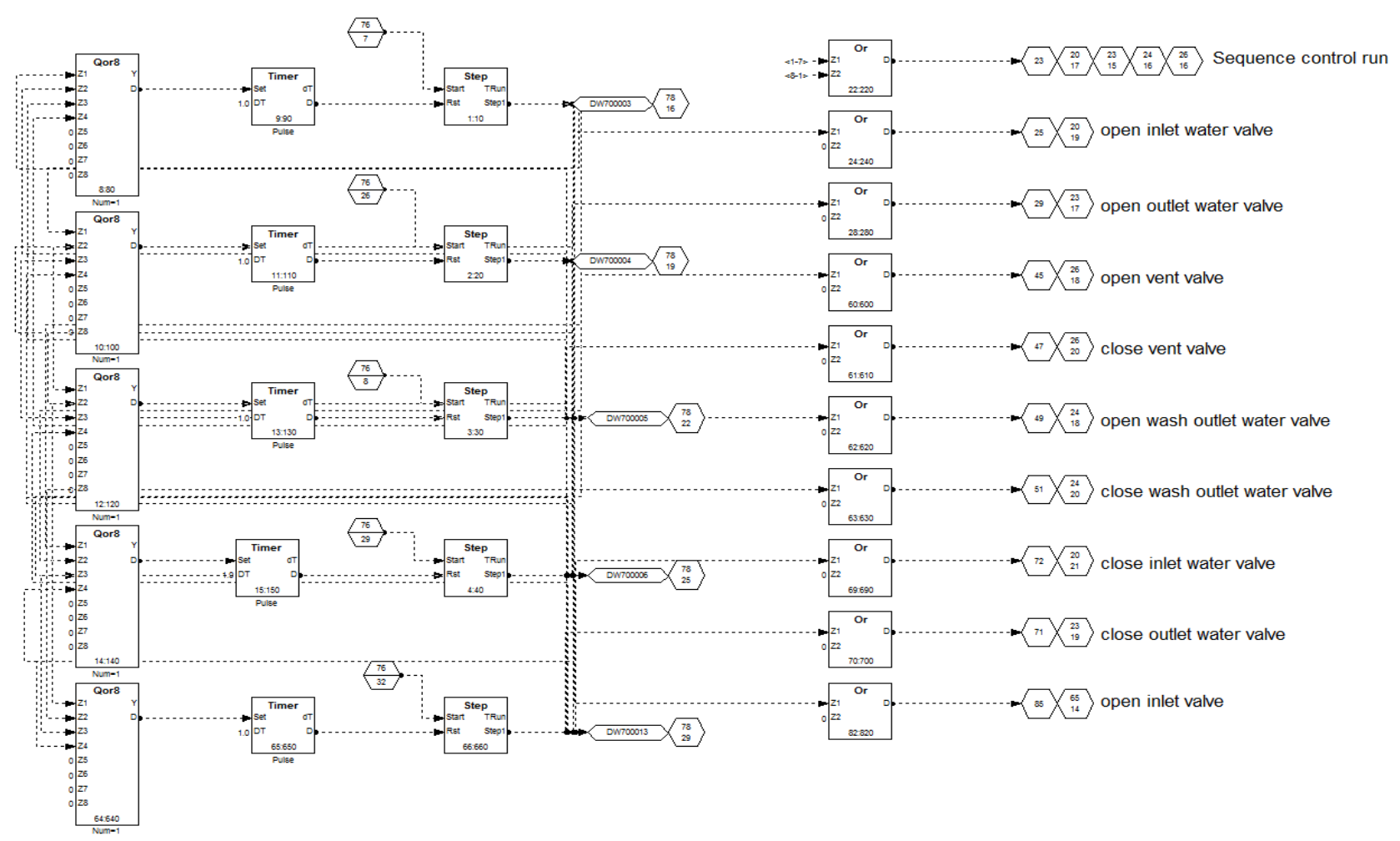

Fig.4. Sequence control step

\section{Conclusion}

After the modification of DCS, the hardware system is stable. Through the optimization of the control logic, the operation safety of the equipment in the chemical water treatment system is considered and realize stable operation of sequence control. The control strategy is simple and easy to be implemented in DCS. So this method has very high application value.

\section{References}

[1] Kamal El-Nahhas. INFLUENCE OF THE PHYSICAL-CHEMICAL FACTORS ON THE RESIDUALS MANAGEMENT FOR DRINKING WATER TREATMENT PLANTS[A]. International Water Technology Association.Sixteenth International Water Technology Conference Proceedings[C].International Water Technology Association:,2012:13.

[2] TorOve Leiknes. The effect of coupling coagulation and flocculation with membrane filtration in water treatment:A review[J]. Journal of Environmental Sciences, 2009,01:8-12.

[3] Xitong Liu,Mengshu Wang,Shujuan Zhang,Bingcai Pan. Application potential of carbon nanotubes in water treatment:A review[J]. Journal of Environmental Sciences,2013,07:1263-1280.

[4] Hrvoje Kusic,Danuta Leszczynska,Natalija Koprivanac,Igor Peterne. Role of quantum dots nanoparticles in the chemical treatment of colored wastewater: Catalysts or additional pollutants[J]. Journal of Environmental Sciences,2011,09:1479-1485.

[5] Treatment of oilfield produced water by anaerobic process coupled with micro-electrolysis[J]. Journal of Environmental Sciences,2010,12:1875-1882. 